\title{
Sunitinib side effects as surrogate biomarkers of efficacy
}

\author{
Christian Kollmannsberger, MD
}

Department of Medicine, University of British Columbia, BC Cancer Agency, Vancouver, BC, Canada

Cite as: Can Urol Assoc J 2016;10(1 1-12Supp17):S245-7. http://dx.doi.org/10.5489/cuaj.4315

\section{Abstract}

With the proliferation of treatment options for the management of metastatic renal cell carcinoma (mRCC) over the past decade, predictive markers of response to therapy are becoming increasingly important. Sunitinib is commonly used in the first-line treatment of mRCC. Common mechanism-based adverse events, including hypertension, hypothyroidism, hand-foot syndrome, and neutropenia, have been explored as potential biomarkers of the clinical efficacy of sunitinib in $\mathrm{mRCC}$ and are reviewed in this article.

\section{Introduction}

Over the past decade, agents that target vascular endothelial growth factor (VEGF) and its receptors have become a standard of care for the treatment of metastatic renal cell carcinoma (mRCC). The selection and sequencing of targeted treatment are driven largely by baseline prognostic factors, with patients classified into poor, intermediate, and favourable risk groups. ${ }^{1,2}$ However, these risk categories are prognostic and not predictive, prompting the exploration of efficacy biomarkers that can be used during treatment to adjust prognosis as needed. Several potential serum, radiological, and tissue-based biomarkers have been evaluated for various agents; ${ }^{3-9}$ however, none has been validated for clinical use in RCC. ${ }^{10}$ As a practical alternative, on-treatment predictors of efficacy have emerged that focus on mechanism-based adverse events (AEs) that reflect the targeted effects of a molecularly targeted agent and its inhibition of a particular pathway.

The anti-VEGFR tyrosine kinase inhibitor (TKI) sunitinib has been available in Canada for the first-line treatment of mRCC since 2006. With more than 10 years of experience with this agent, there is increased understanding of the mechanism-based AEs associated with sunitinib, the most common of which are hypertension, hypothyroidism, hand-foot syndrome, asthenia/fatigue, neutropenia, and thrombocytopenia. These AEs can lead to dose reductions, interruptions, and discontinuations ${ }^{11}$ - all of which may negatively impact outcomes in patients with mRCC. However, some, including hypertension, hypothyroidism, hand-foot syndrome, and neutropenia, have been explored as potential biomarkers of the clinical efficacy of sunitinib. Associations between the onset of toxicity and outcomes have been described with other targeted agents, including the skin toxicity associated with EGFR therapy in colorectal cancer $^{12}$ or pneumonitis with mTOR inhibitors. ${ }^{13}$ This article reviews some of the mechanism-based AEs and their potential role as biomarkers of efficacy for sunitinib in patients with mRCC.

\section{Hypertension}

Hypertension is a common AE associated with agents that target the VEGF pathway, including sunitinib, bevacizumab, sorafenib, and axitinib. ${ }^{14}$ The molecular mechanisms underlying VEGF inhibitor-induced hypertension are unclear. Proposed mechanisms include endothelial dysfunction and increased vascular resistance due to impaired nitric oxide signalling, reduced prostacyclin production, endothelin-1 (ET-1) upregulation, oxidative stress, and rarefaction. ${ }^{15-17}$ Hypertension occurs in approximately one-third of patients treated with sunitinib. ${ }^{18}$ The association between sunitinib-induced hypertension and antitumour efficacy was evaluated in a retrospective analysis of pooled efficacy and safety data from four studies of 4915 patients with mRCC treated with sunitinib $50 \mathrm{mg} /$ day administered on a fourweek-on/two-week-off schedule (four/two). ${ }^{19}$ Hypertension was defined as a maximum systolic blood pressure (SBP) of at least $140 \mathrm{mmHg}$ or a maximum diastolic blood pressure (DBP) of at least $90 \mathrm{mmHg}$. Systolic hypertension was associated with an objective response rate (ORR) of $54.8 \%$, compared with an ORR of $8.7 \%$ in patients without systolic hypertension $(p<0.001)$. Progression-free survival (PFS) $(12.5$ vs. 2.5 months; $p<0.001)$ and overall survival (OS) (30.9 vs. 7.2 months; $p<0.001$ ) were also significantly higher in patients with systolic hypertension than in those without. 
Similar correlations were seen between diastolic hypertension and efficacy. In this retrospective analysis of nearly 5000 sunitinib-treated patients with mRCC, the incidence of hypertension-associated cardiovascular, cerebrovascular, ocular, and renal AEs was low. ${ }^{19}$ Comparable results have been observed in other studies and other VEGFR-TKIs. ${ }^{20-22}$ Donskov et al demonstrated that hypertension and neutropenia influenced outcomes in each IMDC group. ${ }^{20}$ Although not all patients required hypertension to gain a clinical benefit from sunitinib, these results support the hypothesis that hypertension may be a viable biomarker of antitumour efficacy in patients with mRCC and may be used to adjust prognosis during first-line therapy. Treatment of hypertension should follow the regular hypertension guidelines. ${ }^{23}$ Importantly, use of antihypertensive medications does not reduce the antitumour activity of sunitinib. ${ }^{19}$ In one small prospective study, patients undergoing treatment with sunitinib for mRCC underwent aggressive blood pressure monitoring and algorithmic treatment for hypertension according to European guidelines rather than common toxicity criteria. ${ }^{24}$ Nine of the 10 patients were able to achieve uninterrupted, full-dose sunitinib treatment. Such a management approach could complement the use of hypertension as a biomarker - maximizing the therapeutic benefits of sunitinib while minimizing the risk of hypertension-associated complications. Some studies even indicate that the type of antihypertensive treatment may also have an influence on outcomes..$^{25}$

\section{Hypothyroidism}

Hypothyroidism is a common AE associated with sunitinib and other agents in this class. ${ }^{26}$ The potential role of hypothyroidism as a predictive marker of outcomes has been explored. In a prospective analysis of 87 consecutive patients with mRCC treated with sunitinib or sorafenib, subclinical hypothyroidism during treatment was associated with a significant increase in the rate of objective remission compared with euthyroid patients $(28.3 \%$ vs. $3.3 \%$; $p<0.001)$, as well as an increase in the median duration of survival (not reached vs. 13.9 months; $p=0.016$ ).$^{27}$ In a meta-analysis of 11 retrospective and prospective studies of 500 patients treated with sunitinib or sorafenib for $\mathrm{mRCC}^{28}$ there was no significant difference in PFS between patients who acquired hypothyroidism during sunitinib treatment and those who did not. OS was longer in patients who developed hypothyroidism during sunitinib therapy compared with patients who did not (hazard ratio [HR] 0.52; $p=0.01$ ); however, the authors urged caution in interpreting these results due to the retrospective nature of the study.

\section{Other mechanism-based AEs}

Other mechanism-based AEs secondary to VEGRF inhibitors have been studied as potential biomarkers of treatment efficacy. Sunitinib induces neutropenia and thrombocytopenia in approximately $20 \%$ of non-Asian patients and hand-foot syndrome in approximately $30 \%$ of patients. ${ }^{29}$ A pooled retrospective analysis of patients from five prospective clinical trials evaluated the development of hypertension, neutropenia, thrombocytopenia, hand-foot syndrome, and asthenia/fatigue during treatment with sunitinib for mRCC as predictors of PFS and OS. ${ }^{20}$ Neutropenia was associated with significantly longer PFS and OS $(p=0.013$ and $p=0.0122$, respectively), while hypertension and hand-foot syndrome were associated with significantly longer OS ( $p=0.004$ and $\mathrm{p}=0.022$, respectively). In a multivariate analysis, hypertension, neutropenia and hand-foot syndrome remained as independent prognostic factors of OS.

Recently, an Italian centre evaluated the prognostic role of cumulative toxicity in 104 patients with mRCC treated with first-line sunitinib or pazopanib. ${ }^{30}$ Cumulative toxicity - defined as having more than one selected AE of any grade - was associated with a significantly greater median OS (61.2 months vs. 18.7 months; HR 0.23; $\mathrm{p}<0.001)$ and PFS (27.6 vs. 7.2 months; HR 0.31; $p<0.001)$ compared with those who experienced one or no AEs. In this analysis, both OS and PFS were significantly higher in those who experienced hypertension, hypothyroidism, and hand-foot syndrome while on treatment compared with those who did not experience these AEs.

\section{Maximizing quality of life in patients undergoing treatment with sunitinib}

Higher exposure of sunitinib has been shown to correlate significantly with a higher probability of overall response rate, longer time to progression, and increased OS. ${ }^{31}$ Although several frequently occurring AEs of sunitinib, including hypertension, hypothyroidism, neutropenia, and hand-foot syndrome are potentially associated with better clinical outcomes, these AEs can affect patient quality of life, leading to dose reduction or even discontinuation, which may affect patient outcome. Schedule changes, e.g., a two-week-on/ one-week-off schedule, are in many cases a good choice to avoid dose reduction. Early effective management of these AEs is vital to maximizing the patient's quality of life and time on treatment.

\section{Conclusions}

mRCC is a heterogeneous disease, and optimal management is driven largely by the patient's prognosis. Reliable and 
clinically validated predictive biomarkers for response to antiangiogenic therapy are still lacking. The ideal biomarker would be available prior to treatment start, simple, easy to measure, and affordable. To date, treatment-induced hypertension has been the best studied on-therapy biomarker, and is relatively easy to manage. Prospective trials are needed to validate this and other mechanism-based AEs as biomarkers for efficacy.

\section{References}

1. Motzer RJ, Bacik J, Schwartz LH, et al. Prognostic factors for survival in previously treated patients with metastatic renal cell carcinoma. J Clin Oncol 2004;22:454-63. https://doi.org/10.1200/ JC0.2004.06.132

2. Heng DY, Xie W, Regan MM, et al. Prognostic factors for overall survival in patients with metastatic renal cell carcinoma treated with vascular endothelial growth factor-targeted agents: Results from a large, multicentre study. J Clin Oncol 2009;27:5794-9. https://doi.org/10.1200/JC0.2008.21.4809

3. Schneider BP, Wang M, Radovich $M$, et al. Association of vascular endothelial growth factor and vascular endothelial growth factor receptor-2 genetic polymorphisms with outcome in a trial of paclitaxel compared with paditaxel plus bevacizumab in advanced breast cancer: ECOG 2100. J Clin Oncol 2008;26:4672-8. https://doi.org/10.1200/JC0.2008.16.1612

4. Figlin RA, de Souza P, McDermott D, et al. Analysis of PTEN and HIFlalpha and correlation with efficacy in patients with advanced renal cell carcinoma treated with temsirolimus vs. interferon-alpha. Cancer 2009;115:3651-60. https://doi.org/10.1002/cncr.24438

5. Escudier B, Loomis A, Kaprin A, et al. 7103 ORAL association of single nucleotide polymorphisms (SNPS) in VEGF pathway genes with progression-free survival (PFS) and blood pressure (BP) in metastatic renal cell carcinoma (mRCC) in the phase 3 trial of axitinib vs. sorafenib (AXIS Trial). Eur J Cancer 2011;47:S505. https://doi.org/10.1016/S0959-8049(11)72018-4

6. Garcia-Donas J, Esteban E, Leandro-García $\mathrm{L}$, et al. Single nucleotide polymorphism associations with response and toxic effects in patients with advanced renal cell carcinoma treated with first-line sunitinib: A multicentre, observational, prospective study. Lancet Oncol 2011;12:1143-50. https://doi. org/10.1016/S1470-2045(11)70266-2

7. Tran HT, Liu Y, Zurita AJ, et al. Prognostic or predictive plasma cytokines and angiogenic factors for patients treated with pazopanib for metastatic renal-cell cancer: A retrospective analysis of phase 2 and phase 3 trials. Lancet Oncol 2012;13:827-37. https://doi.org/10.1016/S1470-2045(12)70241-3

8. Harmon CS, Deprimo SE, Figlin RA, et al. Circulating proteins as potential biomarkers of sunitinib and interferon-a efficacy in treatment-naive patients with metastatic renal cell carcinoma. Cancer Chemother Pharmacol 2014;73:151-61. hittps://doi.org/10.1007/s00280-013-2333-4

9. Mains JR, Donskov F, Pedersen EM, et al. Dynamic contrast-enhanced computed tomography as a potential biomarker in patients with metastatic renal cell carcinoma: Preliminary results from the Danish Renal Cancer Group Study-1. Invest Radiol 2014;49:601-7. https://doi.org/10.1097/RLI.0000000000000058

10. Jain RK, Duda DG, Willett CG, et al. Biomarkers of response and resistance to antiangiogenic therapy. Nat Rev Clin Oncol 2009;6:327-38. htrps://doi.org/10.1038/nrclinonc.2009.63

11. Oh WK, McDermott D, Porta C, et al. Angiogenesis inhibitor therapies for advanced renal cell carcinoma: Toxicity and treatment patterns in clinical practice from a global medical chart review. Int I Oncol 2014;44:5-16

12. Saltz LB, Meropol NJ, Loehrer PJ Sr, et al. Phase 2 trial of cetuximab in patients with refractory colorectal cancer that expresses the epidermal growth factor receptor. J Clin Oncol 2004;22:1201-8. https://doi. org/10.1200/JC0.2004.10.182

13. Atkinson BJ, Pharm D, Cauley DH, et al. mTOR inhibitor-associated non-infectious pneumonitis in patients with renal cell cancer: Management, predictors, and outcomes. BJU Int 2014;113:376-82. https://doi.org/10.1111/bju.12420
14. Launay-Vacher V, Deray G. Hypertension and proteinuria: A class-effect of antiangiogenic therapies. Anticancer Drugs 2009;20:81-2. https://doi.org/10.1097/CAD.0b013e3283161012

15. Yang $R$, Thomas $G R$, Bunting $S$, et al. Effects of vascular endothelial growth factor on hemodynamics and cardiac performance. J Cardiovasc Pharmacol 1996;27:838-44. https://doi.org/10.1097/00005344199606000-00011

16. Wei W, Jin $\mathrm{H}$, Chen ZW, et al. Vascular endothelial growth factor-induced nitric oxide- and PGI2-dependent relaxation in human internal mammary arteries: A comparative study with KDR and Flt-l selective mutants. J Cardiovasc Pharmacol 2004;44:615-21. https://doi.org/10.1097/00005344-20041 1000-00016

17. Steeghs N, Gelderblom H, Roodt J0, et al. Hypertension and rarefaction during treatment with telatinib, a small molecule angiogenesis inhibitor. Clin Cancer Res 2008;14:3470-6. https://doi. org/10.1158/1078-0432.CCR-07-5050

18. Motzer RJ, Hutson TE, Tomczak P, et al. Sunitinib vs. interferon alfa in metastatic renal cell carcinoma. N Engl J Med 2007;356:115-24. https://doi.org/10.1056/NEJMoc065044

19. Rini BI, Cohen DP, Lu DR, et al. Hypertension as a biomarker of efficacy in patients with metastatic renal cell carcinoma treated with sunitinib. J Natt Cancer Inst 2011;103:763-73. https://doi.org/10.1093/ inci/dirl 28

20. Donskov F, Michaelson MD, Puzanov I, et al. Sunitinib-associated hypertension and neutropenia as efficacy biomarkers in metastatic renal cell carcinoma patients. Br J Cancer 2015;113:1571-80. https://doi.org/10.1038/bic.2015.368

21. Rautiola J, Donskov F, Peltola K, et al. Sunitinib-induced hypertension, neutropenia, and thrombocytopenia as predictors of good prognosis in patients with metastatic renal cell carcinoma. BJU Int 2016;117:110-7. https://doi.org/10.1111/bju.12940

22. Rini BI, Schiller JH, Fruehauf JP, et al. Diastolic blood pressure as a biomarker of axitinib efficacy in solid tumours. Clin Cancer Res 2011;17:3841-9. https://doi.org/10.1158/1078-0432.CCR-10-2806

23. Maitland ML, Bakris GL, Black HR, et al. Initial assessment, surveillance, and management of blood pressure in patients receiving vascular endothelial growth factor signaling pathway inhibitors. J Natl Cancer Inst 2010;102:596-604. https://doi.org/10.1093/inci/diq091

24. Bamias $A$, Lainakis $G$, Manios $E$, et al. Diagnosis and management of hypertension in advanced renal cell carcinoma: Prospective evaluation of an algorithm in patients treated with sunitinib. J Chemother 2009;21:347-50. https://doi.org/10.1179/joc.2009.21.3.347

25. Izzedine H, Derosa L, Le Teuff $G$, et al. Hypertension and angiotensin system inhibitors: Impact on outcome in sunitinib-treated patients for metastatic renal cell carcinoma. Ann Oncol 2015;26:1128-33. https://doi.org/10.1093/annonc/mdv147

26. Funakoshi T, Shimada YJ. Risk of hypothyroidism in patients with cancer treated with sunitinib: A systematic review and meta-analysis. Acta Oncol 2013;52:691-702. https://doi.org/10.3109/02841 86X.2012.752579

27. Schmidinger $M$, Vogl UM, Bojic $M$, et al. Hypothyroidism in patients with renal cell carcinoma: Blessing or curse? Cancer 2011;117:534-44. https://doi.org/10.1002/cncr.25422

28. Nearchou A, Valachis $A$, Lind $P$, et al. Acquired hypothyroidism as a predictive marker of outcome in patients with metastatic renal cell carcinoma treated with tyrosine kinase inhibitors: A literature-based meta-analysis. Clin Genitourin Cancer 2015;13:280-6. https://doi.org/10.1016/i.clgc.2014.10.002

29. Kollmannsberger $C$, Bjarnason $G$, Burnett $P$, et al. Sunitinib in metastatic renal cell carcinoma: Recommendations for management of non-cardiovascular toxicities. Oncologist 2011;16:543-53. https://doi.org/10.1634/theoncologist.2010-0263

30. lacovelli R, Verri E, Cossu Rocca M, et al. Prognostic role of the cumulative toxicity in patients affected by metastatic renal cells carcinoma and treated with first-line tyrosine kinase inhibitors. Anti-Cancer Drugs 2016 (Epub before print). https://doi.org/10.1097/CAD.0000000000000439

31. Houk BE, Bello CL, Poland B, et al. Relationship between exposure to sunitinib and efficacy and tolerability endpoints in patients with cancer: Results of a pharmacokinetic/pharmacodynamic meta-analysis. Cancer Chemother Pharmacol 2010;66:357-71. https://doi.org/10.1007/s00280-009-1170-y 